\title{
Towards A Model of Interaction for Mutual Aware Devices and Everyday Artifacts
}

\author{
Sea Ling ${ }^{1}$, Seng Loke ${ }^{2}$, and Maria Indrawan ${ }^{1}$ \\ 1 Faculty of Information Technology, Monash University, Australia \\ 2 Department of Computer Science and Computer Engineering, La Trobe University, \\ Australia
}

\begin{abstract}
Devices like PDAs, mobile phones and Smartcards can communicate with each other and to exchange information and they should be made mutually aware of each other. For privacy reasons, it is essential for the devices to have different levels of awareness and concealment measures so that each device can control how much it wants to be aware of others and how much it wants to be concealed from others. Other works have modelled and implemented awareness among devices, but none, to our knowledge, has provided a formal grounding to their work. We propose a formal model for mutual aware devices, deriving from an existing work on virtual environment called spatial model of interactions. As proof of concept, we also describe two experimental prototypes based on our model.
\end{abstract}

\section{Introduction}

There is a proliferation of devices of myriad forms today, and everyday objects from furniture, appliances to toys with some communication and computational abilities will continue to find applications and will potentially increase in number. A new generation of electronic appliances or the so-called "Smart Device" or "Information Appliances" were introduced to the market. What distinguishes these smart devices from the conventional appliances is that these smart devices are capable of processing information, animation, video, audio, or some other sensory data as well as establishing communication links with other smart devices. A scenario is one where artefacts within a living room (the furniture, the electronic appliances, the lights, the drapes, windows, etc) become aware of each other and can choose to interact for the purposes of the user. A new device brought home is instantly "noticed" by several other artefacts and assimilated into a distributed system. It might be useful for devices or artefacts to be aware of their surroundings and other interconnected devices and artefacts as services from one device can be provided to other devices and vice versa in an efficient manner. Software infrastructure technologies such as Jini and UPnP aim towards such interconnection among devices, facilitated by underlying technologies such as Bluetooth short-range wireless networking. Such infrastructures already exist in the real world but to the authors' knowledge, none has provided a formal definition for its behaviour. 
This paper proposes a formal model for such mutual awareness behaviour (rather than context awareness, in general) where artefacts can be made aware of each other and establish communication with one another. Given two devices A and B, when both device A and device B share the same physical space (up to a pre-specified limit), these two devices can be made aware of each other or be hidden from each other. The use of concealment and awareness rules in our model provides a mechanism to control a device's awareness towards other devices. By conforming to these rules, one device can be either made aware of another device, hide itself from other devices or be revealed to other devices. Our proposed model will not be restricted to two but cater for many devices. Our model also supports dissemination of a small amount of data about devices, inspired by presence technologies such as instant messaging systems [2]. Apart from the underlying networking and software infrastructure, there are at least two further issues involved. The first is how devices can be aware of one another in a controllable way, i.e. a device ought to have some way to determine how many (and which) other devices (or persons) it wants to be aware of and how many (or which) other devices (or persons) can be made aware of the device. The second issue is: if a device $\mathrm{A}$ is aware of another device B, then what is it about B that device A should be aware of? Devices might broadcast introductory information about themselves for other devices as well as receive such information from others. Some of these issues might be application specific but a more general model will be useful.

Our model is inspired by the spatial model of mutual awareness used in MASSIVE [3] for virtual environments, where entities in a virtual environment are made aware (or unaware) of one another via a spatial model involving notions such as nimbus, focus, and aura surrounding entities. We bring such concepts over to devices and everyday smart objects so that we can speak of the nimbus, focus and aura of artifacts. We also integrate into our model features of presence [2], typically used in instant messaging systems.

There are several models and architectures available for mutual awareness environment. Related research on context awareness was first carried out by [1] which has established itself as the fundamental concepts of mutual awareness. Several distinctive models of context awareness developed over the years are MASSIVE [3], AROMA [5], Context Unity [7] and SOCAM [6]. These models are different in design and implementation to create their own context aware environments, with some using context representation, symbols and/or pure abstraction.

The goal is therefore to create a generic formal model of awareness by extending the spatial model of awareness [3] and presence [2]. In this paper, we first describe the background on context-aware models, providing an overview of the related concepts described in the literature. We also compare and contrast the existing models and architectures mentioned earlier. From the comparison, we identify what is lacking in these models. We then describe our model of mutual awareness and the rules of awareness and concealment. Using two prototype im- 
plementations, we then illustrate how our system is able to enhance and improve upon the existing models.

\section{Modelling Awareness: An Overview}

\subsection{Context Aware Artifacts}

Context-aware artifacts can communicate with other artifacts or human beings depending on the states of the artifacts, as reviewed in [15]. These artifacts could "reach out" towards other artifacts in order to perform other tasks or to inform human beings with certain messages. All of these complicated behaviors could be made possible with one or more sensors. For example, a potential buyer walks into a departmental store and touches a toaster; the toaster would then try to sell itself to that buyer. A more complex behavior would be when an authorized person walks toward a security door; the sensors automatically detect the identity of the person and authorize him by opening the door.

In mobile applications, the challenge of context-aware devices is the ability to connect hosts to exchange data in a dynamic manner as hosts arrive and leave (i.e., the network topology changes constantly). Thus, context-aware devices can be highly adaptive, opportunistic and relies on the resource availability [7]. With the possibility of context-aware behaviors from these devices, a whole new frontier of electronic devices in terms of functionalities that were impossible in the past are made possible.

In our project, we aim to create a system for context aware devices that are capable of entering and leaving the system, allowing devices to have control over what it can and cannot see, the use of metadata to store essential information to preserve privacy and the use of context information to perform more complex operations.

\subsection{Spatial Model of Interaction and Presence}

The Spatial Model of Interaction developed by [?] is used for managing and controlling the information flow in virtual environments. Its core concept is the space within which objects communicate. The way to achieve this is by allowing each object to have its own aura. As described by [9], aura is simply a subtle sensory stimulus of "attraction" that transmits "signals of attraction" governed by the "laws of attraction". Aura is also defined as a "sub-space which effectively bounds the presence of an object within a given medium which acts as an enabler of potential interaction" [8]. Each object has a territory of space that surrounds the object. Information exchange or establishment of connection between the two objects occurs when these territories crossover, making interaction between objects possible within the virtual space.

Objects are able to control the interaction by having degrees in the level of awareness between them. The level of awareness is realised by the concepts of focus and nimbus which define how one object's interaction can be redirected 
towards another object and how much aware it is of one object towards another $[8,10]$. Basically, the more an object is within your focus, the more aware you are of it and the more an object is within your nimbus, the more aware it is of you.

This means that objects can be made aware of other devices by manipulating the nimbus and focus within the shared space. By knowing the degrees of focus and nimbus between devices, awareness of devices can be determined. In our model, we make the degrees of focus and nimbus more concrete by assigning distinct numerical values to the focus and the nimbus of an object. Intuitively, an object $\mathrm{A}$ with a higher focus value over the nimbus value of another object $\mathrm{B}$ will be aware of object B. Alternatively, object A with a higher value of nimbus value over the focus value of $\mathrm{B}$, will not allow $\mathrm{B}$ to be aware of $\mathrm{A}$.

In recent years, instant messaging system has evolved using presence in the wireless and wired computing world. It models another type of awareness to other people based on the person's availability and whether he/she is currently online [2]. According to Nokia [12], presence is a dynamic user profile variable which represents itself towards others and others towards itself. It is also capable of sharing information and provides control services. Such information could be personal, location, contextual, device status and preferred contact method. It was agreed that this concept can be expanded from a simple online/offline description to a much richer presence [12].

Information on the availability, whereabouts, the conditions of user are continuously shared. By providing such knowledge on other users, presence allows users to have their own control on when and how they should be communicating with another user more effectively. However, security and privacy issues must be considered as the information sharing increases.

\subsection{Related Work}

Context-aware devices were initially researched by Schilit et al. [1] whose intention is to make devices aware of other devices, the surrounding environment and to allow communication to take place. In subsequent years, several similar projects on context awareness such as MASSIVE [3], AROMA [5], Context Unity [7] and SOCAM [6] have evolved. Each model is targeted at different application domains such as location tracking, virtual worlds and mobility.

MASSIVE (Model, Architecture and System for Spatial Interaction in Virtual Environments) [8] is a prototype implementation based on the Spatial Model of Interaction in virtual context aware environment. Context UNITY is a model of mutual awareness theory with the presence of mobility extending the work on Mobile UNITY [7]. Mobile UNITY is based on the UNITY model [13] with additional notations for mobility and proof logic. It provides a formal representation for mobility to the context-aware environment to allow reasoning mechanism and behaviour manipulation according to the change of context. The SOCAM (Service Oriented Context-Aware Middleware) project [6] is an architecture for developing context-aware services. The aim of this project is to offer an efficient infrastructure support for context-aware services. AROMA is a model of 
mutual awareness developed by Pedersen and Skoler [5]. This model uses a different approach to MASSIVE. It uses a pure abstract representation for objects, re-mapping on media signals and extending the application domain to include social interactions.

\section{Formalisng Spatial Model with Presence for Mutual Aware Devices}

The objective is to develop a formal model for electronic devices not only able to communicate with each other, but also able to understand and identify its surrounding devices. Thus, we propose a solution by combining the spatial model of interaction with presence from instant messaging system.

We use the principles of spatial model to control the availability of devices for communication and to facilitate the discovery of devices and establishing connections. The aura of each device is assumed to be the area within the range of the communication limit. The idea is that given the focus and nimbus of each device, the level of awareness is able to be controlled and devices can behave differently under different programmable conditions. We use the principle of presence to propose an identification mechanism which is capable of identifying the right devices as well as providing additional "condition" information. By incorporating presence, each device is provided with a metadata to uniquely identify itself and its current condition. The device would be able to change its metadata to inform its change to other devices.

The concepts described in Section 2 are now applied to our model. Nimbus is represented by the range of communication and the level of awareness for each device. Focus is modelled by the level of concealment set for each device. Presence is the metadata containing device information. Every device possess different levels of awareness and concealment. For example, device $\mathrm{X}$ is aware of device $\mathrm{Y}$ (i.e., device $\mathrm{Y}$ is visible) if $\mathrm{X}$ 's awareness level is higher than Y's concealment level. Alternatively, $\mathrm{X}$ is not aware of $\mathrm{Y}$ (i.e., device $\mathrm{Y}$ is invisible) if Y's concealment level is higher than X's awareness level.

We generalise the above for all devices.

Definition 1 (Awareness and Concealment) For every device $i$, let $a_{i}$ be its awareness level and $c_{i}$ be its concealment level.

Given any two devices $x$ and $y$, the following holds:

$-a_{x} \geq c_{y}$ if and only if $x$ is aware of $y$.

$-a_{x}<c_{y}$ if and only if $x$ is not aware of $y$.

We have the following propositions:

Proposition 1 (Mutual Awareness) Given any two devices $x$ and $y, x$ and $y$ are aware of each other if and only if $a_{x} \geq c_{y}$ and $a_{y} \geq c_{x}$.

Proof: By Definition 1. 
Proposition 2 (Mutual Concealment) Given any two devices $x$ and $y, x$ and $y$ are not aware of each other if and only if $c_{x}>a_{y}$ and $c_{y}>a_{x}$.

Proof: By Definition 1.

Proposition 3 Given any two devices $x$ and $y, x$ is aware of $y$ and $y$ is not aware $x$ if and only if $a_{x} \geq c_{y}$ and $c_{x}>a_{y}$.

Proof: By Definition 1.

Proposition 4 Given any three devices $x, y$ and $z$, such that $x$ is aware of $y$ and $y$ is aware of $z, x$ is aware of $z$ if $c_{y} \geq c_{z}$.

Proof: Given $a_{z} \geq c_{y}$ and $a_{y} \geq c_{z}$, if $c_{y} \geq c_{z}$, then the result follows that $a_{x} \geq c_{z}$.

Theorem 1 Given a set of devices $\left\{x^{1}, x^{2}, x^{3}, \cdots, x^{k}, \cdots, x^{n}\right\}$, for each $k(1 \leq$ $k \leq n-1)$, if $a_{x^{k}} \geq c_{x^{k+1}}$ and $c_{x^{k}} \geq c_{x^{k+1}}, x^{k}$ is aware of $x^{n}$.

Proof: For each $k(1 \leq k \leq n-1)$, given $a_{x^{k}} \geq c_{x^{k+1}}, a_{x^{k+1}} \geq c_{x^{k+2}}, \cdots, a_{x^{n-1}} \geq$ $c_{x^{n}}$, if $c_{x^{k+1}} \geq c_{x^{k+2}}, c_{x^{k+2}} \geq c_{x^{k+3}}, \cdots, c_{x^{n-1}} \geq c_{x^{n}}$, then from Proposition 4, $a_{x^{k}} \geq c_{x^{k+2}}, a_{x^{k+1}} \geq c_{x^{k+3}}, \cdots, a_{x^{n-2}} \geq c_{x^{n}}$. Since for all $k$ and $i$ where $i \geq k$, $c_{x^{k}} \geq c_{x^{i}}$ and $a_{x^{k}} \geq c_{x^{i}}, a_{x^{k}} \geq c_{x^{n}}$, i.e., all devices all aware of $x^{n}$.

Proposition 5 Given any three devices $x, y$ and $z$ such that $x$ is aware of $y$ and $y$ is aware of $z, x$ is aware of $z$ if $c_{y} \geq a_{y}$.

Proof: Given $a_{x} \geq c_{y}$ and $a_{y} \geq c_{z}$, if $c_{y} \geq a_{y}$, the result follows that $a_{z} \geq c_{z}$.

Theorem 2 Given a set of devices $\left\{x^{1}, x^{2}, x^{3}, \cdots, x^{k}, \cdots, x^{n}\right\}$, for each $k(1 \leq$ $k \leq n-1)$, if $a_{x^{k}} \geq c_{x^{k+1}}$ and $c_{x^{k}} \geq a_{x^{k}}, x^{k}$ is aware of $x^{n}$.

Proof: If $c_{x^{k}} \geq a_{x^{k}}$ for all $k$, then given $a_{x^{k}} \geq c_{x^{k+1}}$, it follows that $c_{x^{k}} \geq c_{x^{i}}$ for all $i \geq k$. The result then follows from Theorem 1 .

The implication of the above rules is that as long as each device maintains the condition in Theorem 1 or Theorem 2, the awareness relationship will be transitive, regardless of how many devices there are in the environment. Maintaining such a condition might be a "social-mile" imposed on each device in order to have a transitively aware society of devices. Hence, our model allows different situation of mutual (non-)awareness to be represented. Adjustments to the level of mutual (non-)awareness can be done by adjusting the levels of awareness and concealment for each device.

\section{Experimemtal Prototypes}

We describe two experimental prototypes, i.e., proof-of-concept implementations of our model: one for artifacts without computational capabilities (using RFID tagging) and one for devices with computational capabilities (and Bluetooth enabled). While we describe them separately, combinations of the two technologies involves artifacts and computational devices are possible. 


\subsection{RFID}

RFID technology uses tags and readers. The technology has an advantage of simulating a more complex device through programming techniques whilst keeping the focus on developing a model based on context awareness. Devices that use this technology are mostly keycards, key rings, or tags that normally do not possess processing and storage capability.

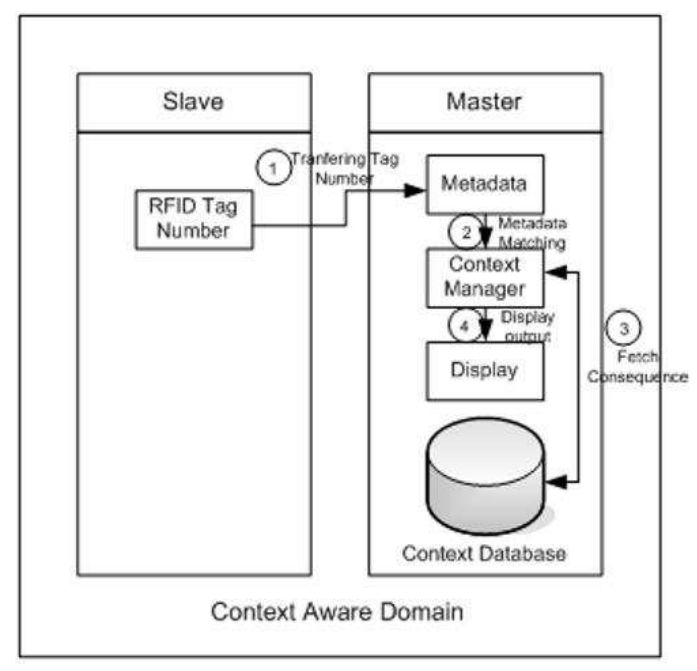

Fig. 1. RFID Implementation

Figure 1 illustrates the architecture consisting of a master and a slave. The figure uses multidirectional and bidirectional arrows to indicate the flow of information and actions throughout the simple system. There are four top level steps for this model. When the RFID tag of the slave is scanned and the master is able to "find" the slave device based on the rules of awareness and concealment, the tag number of the slave device is transferred to the master device (Step 1). Metadata contains information regarding the slave devices including their tag ids and the awareness and concealment levels. The information is stored in the master device.

Context Manager is the software component that deals with the discovery of new slave devices entering and leaving the boundaries of the master device. It also deals with generating context actions based on the information fetched from the Context Database which is data repository containing information about slave devices and the consequences or actions that corresponds to the context information. A list of consequences is stored in the database and the retrieval of these consequences is based on the matching of context information provided by the slave devices. 


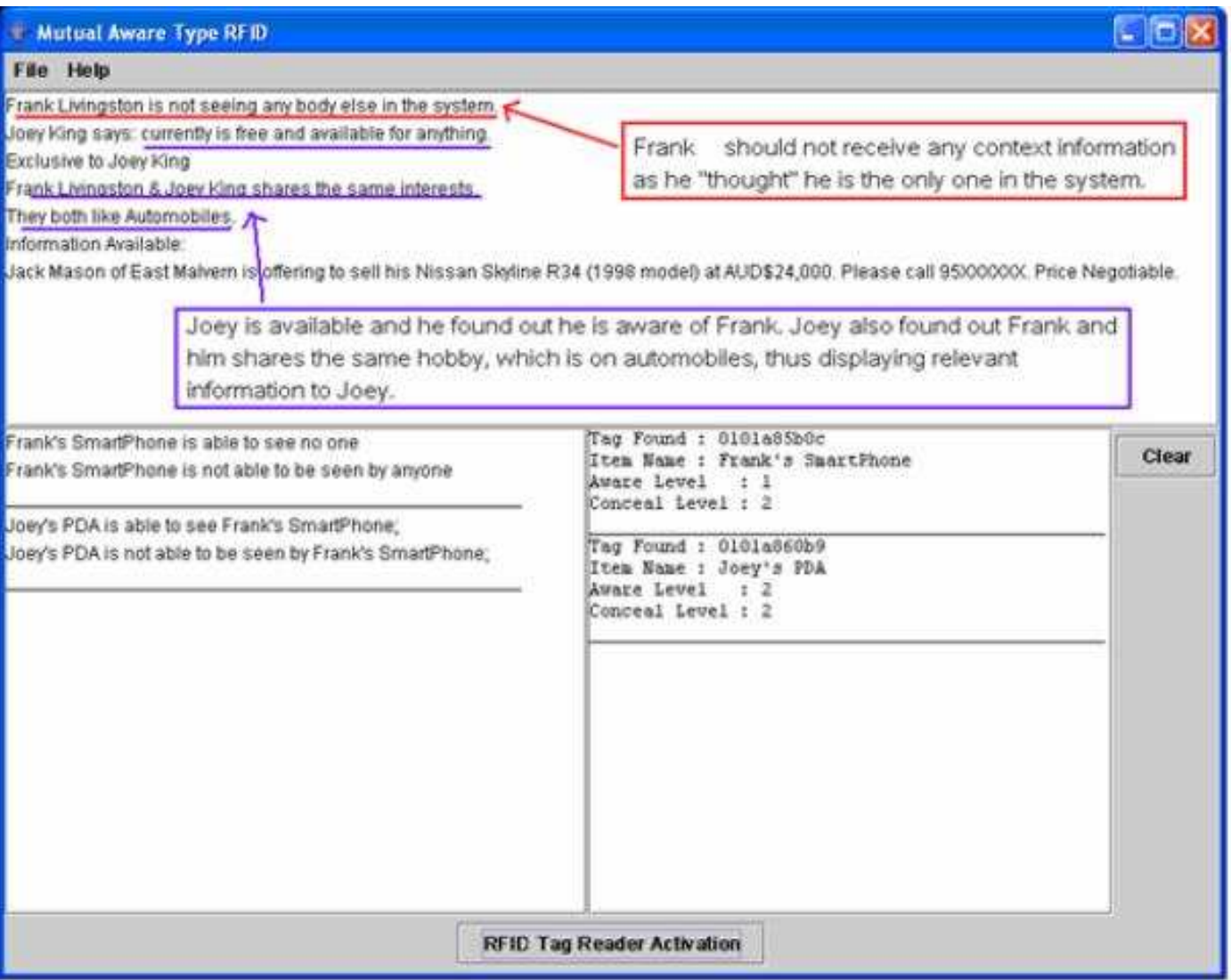

Fig. 2. Sample Screenshot

In Step 2 and Step 3, the Context Manager detects any incoming slave devices based on the rules of Awareness and Concealment. The slave device's metadata (Presence information) is then collected and processed. The processed data is then used as query to the Context Database to retrieve any relevant information regarding the slave device and provide a list of possible actions to be taken. The forth and final step is the display of what actions both the master device and slave device take.

In the prototype, for demonstration, we used the unique RFID tag 0101a85b0c to represent a SmartPhone, belonging to a person called Frank Livingston and we use tag 0101a860b9 to represent a PDA belonging to a person called Joey King. The awareness and concealment levels of Frank's SmartPhone and Joey's PDA are as follow:

- Frank's SmartPhone: Awareness level = 1, Concealment level = 2 


\section{- Joey's PDA: Awareness Level $=2$ Concealment Level $=2$}

Figure 2 is a screenshot of the prototype with the above information displayed in the bottom right frame of the window. The result based on the awareness and concealment rules is displayed on the bottom left frame of the window.

Once devices are aware of each other, they can exchange context information. By using the mutual awareness as the foundation for the prototype, additional functionalities can be added into the system. In the top frame of the window in Figure 3, examples of possible context information are displayed based on the devices' status. In the prototype, such information is retrieved from the metadata by the Context Manager.

This context information exchange feature can be made much richer for future development, where users can actually send or store information to perform a variety of operations. For example, in the commercial world, when a person carrying a PDA that contains a shopping list starts walking, his/her PDA will automatically beep or vibrate when he/she is near a supermarket, indicating that the supermarket has the items the user wants to purchase.

\subsection{Bluetooth}

Another way to implement the model of mutual aware artefacts is to use Bluetooth $^{3}$ technology as the communication medium for devices with processing and storage capability. The nature of this technology enables the discovery of devices easily, and we have considered how this can be exploited in our model. Bluetooth devices are capable of discovering other Bluetooth devices when they are within range of each other. In the context aware manner, the discovery of these devices is not necessarily part of the system. The only way the device can be recognized by the system is through the recognition of device IDs provided in the metadata and only if it passes the rules of Awareness and Concealment as given in Section 3. Metadata is a file that stores all the essential information of a particular device which includes information such as device ID, awareness and concealment levels, device owner, contextual information, etc.

In this case study, we have:

- Master device:

- Desktop PC in Jack's office at Awareness level $=3$ and Concealment level $=2$.

- Slave devices:

- Jack's PDA at Awareness level $=2$ and Concealment level $=3$.

- Matt's PDA at Awareness level $=1$ and Concealment level $=1$.

- Miscellaneous device: Jack's SmartLock on his office door.

\section{The Scenario:}

Jack is a lecturer at a university and Matt is one of Jack's student. Matt has

\footnotetext{
${ }^{3}$ More information on Bluetooth can be found in Bluetooth Special Interest Group at www.bluetooth.com
} 
an appointment coming up with Jack in Jack's office in a few minutes. However, Jack is late and he is not in his office. He did however manage to leave a message to Jack on his desktop PC.

\section{Situations:}

1. Matt arrives at Jack's office.

2. Matt's PDA beeps and was told from Jack's Desktop PC that Jack is late and will arrive shortly.

3. Matt tries to reply but he could not find Jack's Desktop PC.

4. A few minutes later, Jack arrives at his office.

5. Jack's PDA beeps and was informed that Matt has received his message.

6. Jack and Matt meet up in front of Jack's office.

7. Jack then accesses his Desktop PC through his PDA and opens the SmartLock on his office door.

By conforming to the rules, Matt's PDA has level 1 Awareness and is not aware of the Desktop PC existence but the Desktop PC is still able to see Matt's PDA and sends him Jack's message. Jack's PDA has level 2 Awareness and he can access the Desktop PC and unlocks the door.

An application that performed the above functions has been developed based on the proposed model. Additional contextual information can be added into the application, e.g. GPS (Global Positioning System) integrated PDA can use location as part of contextual information and relay that information to other devices when required.

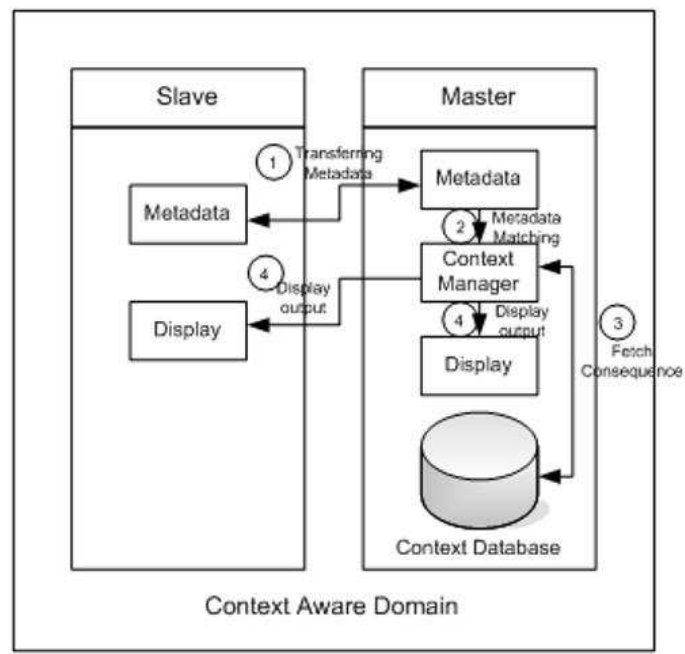

Fig. 3. Bluetooth Implementation 
The mutual aware artefacts system on Bluetooth consists of the master and the slave shown in Figure 3. The previous implementation using RFID technology can also be converted to Bluetooth platform without much modification. There is however one key difference. The slave now consists of a screen for displaying details and also storing its own metadata. The metadata stored on slave devices eliminates the need for the master device to have a metadata collection as in the RFID technology example. With RFID technology, the tagged slaves might not have computational/storage capabilities. Thus, it might not be able to store metadata, thereby requiring the master device to store such metadata. With the Bluetooth technology, the slaves are assumed to have computational/storage capabilities to store metadata describing themselves. The processing of metadata and the fetching of consequences is the same as in the RFID technology except the actions are sent back to the slaves and displayed there.

\section{Conclusion}

We have proposed a model for mutual aware artifacts by combining a Spatial Model of Awareness [8] with Presence [2]. The model includes the mechanism to control the awareness levels of devices using the Rules of Awareness and Concealment. We have also adapted ideas from Instant Messaging Systems by storing presence data in metadata form. The model can be used as the fundamental layer for context aware systems where devices are made aware of one another. By only exposing essential information of a device to other devices, the model preserves the devices' privacy and enables the levels of awareness and concealment to be changeable (e.g. at runtime). Two prototypes are also presented based on RFID tags and Bluetooth technology. They serve to check the validity of the proposed model to handle mutual awareness between multiple devices.

Other possible extensions of the proposed model of awareness are:

1. Intelligent Mobile Agents

The integration with intelligent mobile agents will enable the mutual awareness capability to further enhance agents' capabilities.

2. Context Systems with Artificial Intelligence

The implementation of large scale context aware system with the assistance of intelligent searching would greatly enhance the output choices.

For a more realistic application, we are currently extending this model to enable location tracking. The system implementation utilises the Ekahau positioning engine. While tracking the locations of devices, we are investigating what it means to have different mobile device auras to collide in the spatial model. Initial results suggest that the model can be further enhanced to reflect different levels of granularity in representing interaction and services.

Location technologies tend to have inaccuracies so that an object is represented as being within a (perhaps distorted) circle of a particular radius (from several centimetres to a few metres depending on the technology). One can interpret this "circle" as the aura of the object and exploited for mutual object pre- 
interaction (e.g., preparations on the objects before actual message exchanges once overlapping of aura (or "circles") is detected).

\section{References}

1. Schilit, B. N., Adams, N, Want, R. (1994) Context-Aware Computing Application. Proceedings of the Workshop on Mobile Computing Systems and Applications, Santa Cruz, CA, December 1994, IEEE Computer Society: 85-90.

2. Vogiazou, Y. (2002) Wireless Presence and Instant Messaging. [WWW] Available: http ://www.jisc.ac.uk/index.cfm?name = techwatch_report_0207 Accessed: 18 September 2006.

3. Greenhalgh, C. and Benford, S. (1995) MASSIVE: A Collaborative Virtual Environment for Teleconferencing. ACM Transactions on Computer-Human Interaction. 2(3):239-261.

4. Greene, D., O'Mahony, D. (2004) Instant Messaging and Presence Management in Mobile Ad-Hoc Networks. 2nd IEEE Conference on Pervasive Computing and Communications Workshops, PerCom 2004 Workshops, pp. 55-59.

5. Pedersen, E, R., Sokoler, T. (1997) AROMA: Abstract Representation of Presence Supporting Mutual Awareness. Conference on Human Factors in Computing Systems, Proceedings of the Special Interest Group on Computer-Human Interaction (SIGCHI) conference on Human factors in computing systems.

6. Gu, T., Pung, H.K. and Zhang, D.Q. (2004) Toward an OSGi-based infrastructure for context-aware applications. IEEE Pervasive Computing, 3(4), Oct-Dec 2004: 66-74.

7. Julien, C., Payton, J., Roman, G, C. (2004) Reasoning About Context-Awareness in the Presence of Mobility. Electronic Notes in Theoretical Computer Science, Volume 97, 22 July 2004: 259-276.

8. Benford, S., Bowers, J., Fahlen, L, E., Greenhalgh, C. (1994) Managing Mutual Awareness in Collaborative Virtual Environments. Proceedings of Virtual Reality Software Technology Conference '94, ACM Press, Singapore.

9. Ferscha, A., Hechinger, M., Mayrhofer, R., Rocha, D, S., Franz, M., Oberhauser, R. (2004) Digital Aura. Pervasive Computing, Second International Conference, Pervasive 2004, Vienna.

10. Rodden, T. (1996) Populating the Application: A Model of Awareness for Cooperative Applications. Proceedings 1996 ACM Conference on Computer Supported Cooperative Work (CSCW'96), 87-96.

11. Herrero, P., Antonio, D, A. (2002) A Human Based Perception Model For Cooperative Intelligent Virtual Agents. CoopIS/DOA/ODBASE 2002. pp. 195-212.

12. Nokia. Staying in touch with presence, white paper. [WWW] Available: http : //www.nokia.com/BaseProject/Sites/NOKIA_MAIN_18022/CDA/Categories/ Networks/Technologies/MessagingandPresence/PresenceandInstantMessaging/ _Content/_Static_Files/presence_a4_0711.pdf Accessed: 18th September 2006

13. Chandy, K. M. and Misra, J. (1988) Parallel Program Design: A Foundation. Addison-Wesley, NY, USA.

14. Fontana, J. (2004) Presence applications poised for takeoff. [WWW] Available: http : //www.nwfusion.com/news/2004/090604specialfocus.html Accessed: 18th September 2006

15. Loke, S. (2006) Context-Aware Artifacts: Two Development Approaches. IEEE Pervasive Computing, 5(2), Apr-Jun, 2006: 48-53. 